\title{
Antioxidant proteins TSA and PAG interact synergistically with Presenilin to modulate Notch signaling in Drosophila
}

\author{
Michael F. Wangler ${ }^{1,2^{*}}$, Lawrence T. Reiter ${ }^{1,3^{*}}$, Georgianna Zimm ${ }^{1}$, Jennifer Trimble-Morgan ${ }^{1,4}$, Jane $\mathbf{W u}^{5}$, \\ Ethan Bier ${ }^{1} \bowtie$ \\ ${ }^{1}$ Department of Biology, University of California at San Diego, San Diego, CA 92093, USA \\ 2 Present address: Department of Molecular and Human Genetics, Baylor College of Medicine, One Baylor Plaza, Houston, TX \\ 77030, USA \\ ${ }^{3}$ Present address: Department of Neurology, University of Tennessee Health Science Center, Memphis, TN 38163, USA \\ ${ }^{4}$ Present address: Saddleback College, 28000 Marguerite Parkway, Mission Viejo, California 92692, USA \\ ${ }^{5}$ Department of Neurology and Center for Genetic Medicine, Northwestern University, Chicago, IL 60611, USA \\ \ Correspondence: ebier@ucsd.edu \\ Received June 15, 2011 Accepted June 27, 2011
}

\section{ABSTRACT}

Alzheimer's disease (AD) pathogenesis is characterized by senile plaques in the brain and evidence of oxidative damage. Oxidative stress may precede plaque formation in $A D$; however, the link between oxidative damage and plaque formation remains unknown. Presenilins are transmembrane proteins in which mutations lead to accelerated plaque formation and early-onset familial Alzheimer's disease. Presenilins physically interact with two antioxidant enzymes thiol-specific antioxidant (TSA) and proliferation-associated gene (PAG) of the peroxiredoxin family. The functional consequences of these interactions are unclear. In the current study we expressed a presenilin transgene in Drosophila wing and sensory organ precursors of the fly. This caused phenotypes typical of Notch signaling loss-of-function mutations. We found that while expression of TSA or PAG alone produced no phenotype, co-expression of TSA and PAG with presenilin led to an enhanced Notch loss-offunction phenotype. This phenotype was more severe and more penetrant than that caused by the expression of Psn alone. In order to determine whether these phenotypes were indeed affecting Notch signaling, this experiment was performed in a genetic background carrying an activated Notch (Abruptex) allele. The phenotypes were almost completely rescued by this activated Notch allele. These results link peroxiredoxins with the in vivo function of Presenilin, which ultimately connects two key pathogenetic mechanisms in AD, namely, antioxidant activity and plaque formation, and raises the possibility of a role for peroxiredoxin family members in Alzheimer's pathogenesis.

KEYWORDS Presenilin, Alzheimer's disease, peroxiredoxin, Notch

\section{INTRODUCTION}

Alzheimer's disease $(A D)$ is a neurodegenerative disease leading to dementia with a characteristic brain pathology marked by the accumulation of neurofibrillary tangles and senile plaques in the central nervous system (CNS) (Selkoe, 2001). The pathogenesis of $A D$ is the subject of extensive study, but the molecular sequence of events ultimately leading to progressive neurodegeneration remains unclear (Smith et al., 2000; Van Gassen et al., 2000; Perry et al., 2002). Pathologic changes underlying the disease have been explored in post-mortem brain tissue (McLellan et al., 2003; Shan et al., 2003), neuroimaging (Fox and Schott, 2004; Schott et al., 2005), biochemical analyses of presenilins as part of the Y-secretase complex (Wolfe et al., 1999; Zhou et al., 2002), and functional analysis of implicated proteins in model organisms like Drosophila (Greeve et al., 2004; lijima

\footnotetext{
*These authors contributed equally to the work.
} 
et al., 2004; Crowther et al., 2005). It has been suggested that there are two crucial neuropathological features of AD brains, the formation of amyloid plaques and the accumulation of oxidative stress (Lahiri and Greig, 2004). The strongest evidence for the crucial role of amyloidogenesis in $A D$ pathogenesis comes from cases of familial AD (FAD). These early onset Mendelian cases result from mutations in the amyloid precursor protein (APP) (Goate et al., 1991) or mutations in one of the two presenilin genes, PSEN1 and PSEN2 (Levy-Lahad et al., 1995; Rogaev et al., 1995; Sherrington et al., 1995). As part of the $\mathrm{Y}$-secretase protein complex, presenilins are responsible for amyloid cleavage and FAD mutations in PSEN1 and PSEN2 lead to overproduction of the neurotoxic 42 amino acid form of $\beta$-amyloid (Martins et al., 1995; Borchelt et al., 1996; Duff et al., 1996; Scheuner et al., 1996; Citron et al., 1997; Xia et al., 1997; Mehta et al., 1998; Oyama et al., 1998). This human genetic evidence and the amyloid composition of the senile plaques have made the presenilin proteins a major focus of $A D$ research (Haass and De Strooper, 1999).

In addition to aberrant amyloid processing, global oxidative damage plays a key role in the etiology of AD. Evidence for a role of oxidative damage in $A D$ includes increased reactive carbonyl moieties in the brains of patients with $A D$ (Smith et al., 1992), decreased antioxidant activity in mouse models and human AD brain (Gibson et al., 2000; Leutner et al., 2000; Schuessel et al., 2005), the accumulation of lipid peroxidation by-products in the brains and cerebrospinal fluid of patients with $A D$ (Montine et al., 2002), the presence of oxidative damage to RNA in AD brains (Shan et al., 2003), abnormal oxidation of thiol proteases in the hippocampus in AD patients (Marcum et al., 2005), and mitochondrial abnormalities in AD brains (Smith et al., 2000). While both oxidation-reduction and amyloidogenesis are clearly fundamental to understanding $A D$, few studies have explored specific protein interactions using model organisms between the proteins involved in amyloidogenesis and those involved in oxidative stress.

There is clear evidence of a role for oxidative stress in mice with conditional knockout mutations in the Presenilin genes suggesting that these processes are linked (Gu et al., 2008). One possible explanation could involve the fact that presenilin proteins physically interact with a number of other proteins. Presenilins are eight transmembrane spanning proteins principally associated with the endoplasmic reticulum and the plasma membrane (Nowotny et al., 2000) and comprise the enzymatic part of the $y$-secretase complex (Wolfe et al., 1999), responsible for regulated cleavage events that occur within the membrane. Known targets cleaved by $\gamma$-secretase include $\beta$-amyloid and the Notch receptor. In the case of Notch signaling, the $\gamma$-secretase activity of PSENs is activated by ligand binding to the Notch receptor. Intramembrane cleavage of Notch liberates the intracellular domain (ICD), which then translocates to the nucleus where it associates with the Suppressor of Hairless protein to activate transcription of target genes (De Strooper et al., 1999; Struhl and Greenwald, 1999). While presenilins are required for Notch signaling, overexpression of presenilin in Drosophila leads to a phenotype resembling Notch inactivation suggesting that expression of a single component of the complex acts in a dominant negative fashion (Ye et al., 1999). In addition to these $y$-secretase functions, PSENs have been shown to physically interact with proteins involved in cell adhesion, cytoskeletal regulation, Wnt/wingless signaling pathway, intracellular calcium homeostasis, and apoptosis (Van Gassen et al., 2000; van de Hoef et al., 2009). However, in many cases the functional significance of these interactions is not known (Van Gassen et al., 2000). PSEN1 has also been found to physically associate with two antioxidant enzymes TSA and PAG, both of which are peroxiredoxin enzymes involved in the detoxification of hydrogen peroxide (Zhou et al., 2002; Patenaude et al., 2005).

Based on the evidence that both amyloidogenesis and oxidation-reduction are crucial components of $A D$ pathogenesis and the lack of in vivo validation of protein-protein interactions with presenilin, we tested whether the antioxidant proteins TSA and PAG could modify the phenotypic effect of presenilin overexpression in Drosophila. Drosophila is well suited for such an analysis because of the relative ease in characterizing adult phenotypes, the ability to express transgenes in selected tissues using the GAL4-UAS system (Brand and Perrimon 1993), and the fact that Drosophila has already contributed to the study of AD (Greeve et al., 2004; lijima et al., 2004; Crowther et al., 2005).

\section{RESULTS}

\section{Peroxiredoxin enzymes TSA and PAG enhance the Notch inactivation phenotype caused by Psn expression in the wing}

TSA and PAG physically interact with PSEN1 in vitro (Zhou et al., 2002); however the functional significance of this interaction in vivo remains elusive. We therefore turned to Drosophila as a model system to address this question. TSA and PAG share $76 \%$ amino acid homology, and both have been reported to catalyze the reduction of hydrogen peroxide using key cysteine residues and the protein thioredoxin as a cofactor (Fig. 1A). There is close homology between the four peroxiredoxin family members with two active cysteine residues in humans and the four two-cysteine family members in Drosophila (Fig. 1). We employed the Drosophila UAS-GAL4 transgenic expression system (Brand and Perrimon, 1993; Duffy, 2002) to determine whether TSA and PAG could interact with Presenilin in vivo. Interestingly, while Drosophila Psn is required for Notch cleavage and signaling, overexpression of Psn results in Notch loss-of-function phenotypes, possibly caused by a dominant negative effect 


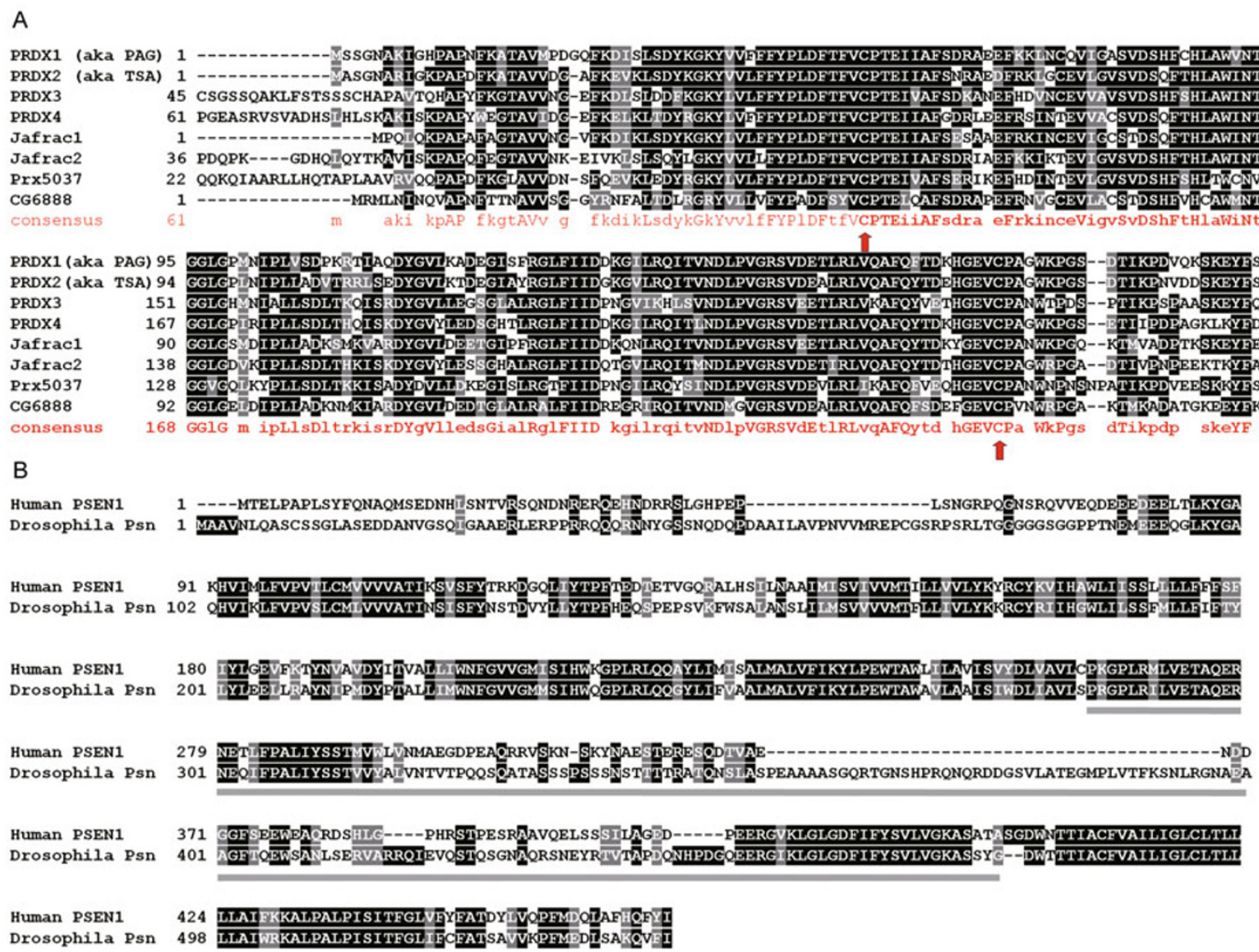

Figure 1. Amino acid sequence alignment of human and Drosophila peroxiredoxin enzymes and Human PSEN1 and Drosophila Psn. (A) Human and Drosophila two-cysteine peroxiredoxin family members are aligned. These proteins catalyze the reduction of hydrogen peroxide to water using a pair of cysteine residues (red arrows) and the thioredoxin protein as a co-factor. Human TSA and PAG share $>76 \%$ amino acid homology, and have been shown to interact with human PSEN1 protein. The four Drosophila two-cysteine family members show close homology to the human versions throughout the length of the proteins (consensus sequence is shown in red). (B) Human PSEN1 and Drosophila Psn are aligned. The Psn transgene shares areas of close homology with PSEN1. The protein-protein interaction with TSA and PAG has been shown to depend on the cytoplasmic loop of the PSEN1 protein between amino acids 265 and 407 (indicated by solid gray line), and this area shows conservation with $P$ sn in distinct regions.

due to the overexpression of Psn (Ye and Fortini, 1999). We explored whether the addition of human TSA and PAG would modulate the phenotype due to overexpression of Drosophila Psn. The site of reported interaction with TSA and PAG, through the cytoplasmic loop region, is a portion of the protein which shows variable homology with distinct areas of strong homology (Fig. 1B, gray bar). We inserted human TSA and PAG coding sequences (Fig. 1) into the Drosophila pUAS expression vector and transformed these constructs into the Drosophila genome. We then expressed TSA and PAG in the developing wing using the MS1096-GAL4 driver (Guillen et al., 1995). The Drosophila wing is a good system for these studies because of its ordered structure with the L1-L5 wing veins (Fig. 2A) that have a well described development through developmental signaling pathways (Bier, 2005). The
MS1096-GAL4 line drives strong GAL4 protein expression in the dorsal region of the wing pouch and weaker expression ventrally. Expression of TSA, PAG, or both with MS1096GAL4 led to no abnormal phenotypes, indicating that strong expression of these peroxiredoxin enzymes alone does not interfere with normal wing development (Fig. 2A). When we expressed the Psn transgene with MS1096-GAL4, we observed a Notch loss-of-function phenotype with thickening of the L3 and L5 wing veins and a significant reduction in the size of the wing surface resulting in dorsal curling of the wings (Fig. 2B). However, when we co-expressed Psn with either TSA or PAG, or with both, we observed a significant increase in the severity of Notch-like phenotypes, most notably vein thickening (Fig. 2C). The wings were also reduced in size and exhibited stronger dorsal curling. 
A

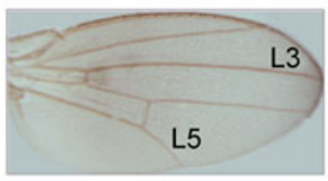

D

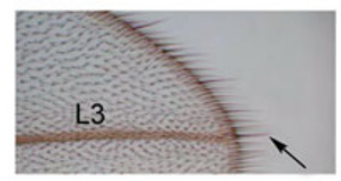

G

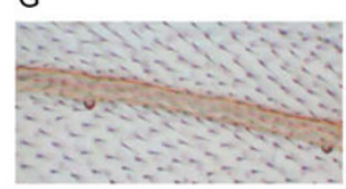

B

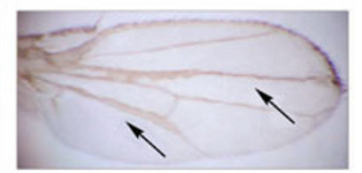

E

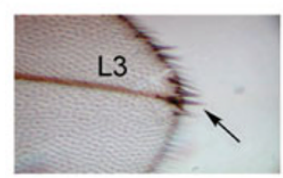

H

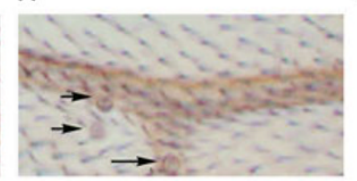

C

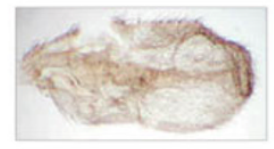

J

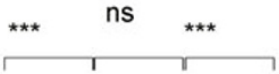

F

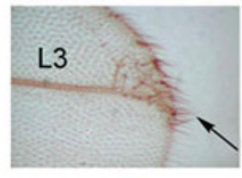

I

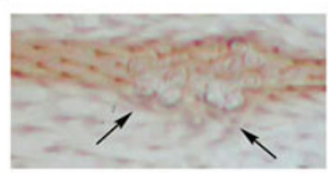

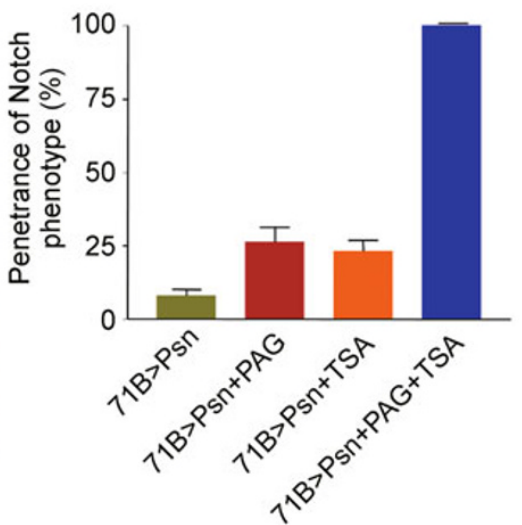

Figure 2. Wing phenotypes of Psn transgene, and Psn in combination with TSA and PAG. (A) MS1096-GAL4 > PAG-UAS; TSA-UAS progeny were indistinguishable from wild type. (B) MS1096-GAL4 > PSn-UAS progeny in contrast exhibited thickening of the L3, and L5 wing veins (arrows). (C) MS1096-GAL4 > Psn-UAS + PAG-UAS + TSA-UAS progeny exhibited severe broadening of the wing veins and reduction in wing size. (D) The 71B- GAL4 > PAG-UAS + TSA-UAS progeny had the wild type pattern of doublerow bristles on the anterior edge of the wing (arrow, wild type is shown). (E) 71B-GAL4 > Psn-UAS progeny exhibited ectopic doublerow bristles at the lateral end of the L3 wing vein (arrow). (F) 71B-GAL4 > Psn-UAS + PAG-UAS + TSA-UAS progeny exhibited more pronounced clusters of ectopic double row bristles at the outer end of the L3 wing vein (arrow). (G) 71B-GAL4 > PAG-UAS + TSAUAS progeny had the wild type pattern of campaniform sensilla, a sensory organ precursor, with regular spacing along the $L 3$ vein (wild type is shown). (H) 71B-GAL4 > Psn-UAS progeny had ectopic campaniform sensilla along the L3 wing vein particularly near the cross-vein (arrows). (I) 71B-GAL4 > Psn-UAS + PAG-UAS + TSA-UAS progeny had marked increase in severity of this phenotype with large clusters of ectopic companiform sensilla present along the L3 vein (arrows). (J) Penetrance of the Notch phenotypes in 71B-GAL4 crosses grouped by genotype. While the Psn genotype had overall penetrance of $8 \%$, the Psn + PAG had a penetrance of $26 \%$, and Psn + TSA had a penetrance of $23 \%$. Finally the Psn + PAG + TSA crosses had a penetrance of $100 \%$ which was significantly higher than the other genotypes.

\section{Peroxiredoxin enzymes TSA and PAG enhance Psn sensory organ precursor phenotypes}

In addition to the effect on the wing veins, we observed similar synergistic effects on the formation of sensory organs when co-expressing these proteins with 71B-GAL4. The 71B-GAL4 line drives moderate levels of GAL4 protein expression ubiquitously in regions of the wing anterior to the L5 vein (Guillen et al., 1995). Expression of the transgenes TSA or PAG with 71B-GAL4 did not alter the wild type pattern of double row bristles (Fig. 2D) or campaniform sensilla (Fig. 2G) along the $L 3$ wing vein. When we expressed UAS$P s n$ with the 71B-GAL4 driver, we observed a modest number of ectopic sensory organs in a fraction of progeny, such as supernumerary double row bristles along the wing margin at the junction with the L3 wing vein (Fig. 2E) and extra campaniform sensilla along the L3 vein (Fig. $2 \mathrm{H}$ ), both Notch loss-of-function phenotypes. Co-expression of TSA and PAG with Psn greatly increased the severity of ectopic clusters of both double-row bristles (Fig. 2F) and campaniform sensilla
(Fig. 2I).

Another indicator of the genetic interaction between Psn and peroxiredoxins was an increase in the penetrance of Notch loss-of-function phenotypes defined as the presence of any ectopic campaniform sensilla or double-row bristle in the 71B-GAL4 progeny. Expression of Psn alone resulted in only partial low-frequency penetrance, which was incrementally enhanced by co-expression with either TSA or PAG and was made fully penetrant if both TSA and PAG were co-expressed with Psn (Fig. 2J).

We also observed Notch loss-of-function phenotypes with ectopic sensory organs in other locations, other than the wing, when Psn was co-expressed with PAG or TSA. Ectopic bristles were observed anterior to the eye using the A9-GAL4 driver. A9 is a low-expressing variant of the MS1096-GAL4 line (Guillen et al., 1995). Expression of TSA plus PAG resulted in no obvious phenotype (Fig. 3A, wild type shown), while expression of $P s n$ alone produced a few ectopic bristles anterior to the eye (Fig. 3B, arrows). In contrast, when Psn was co-expressed with PAG plus TSA, the number of these 
A

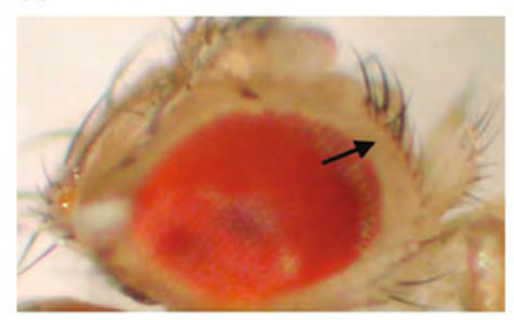

D

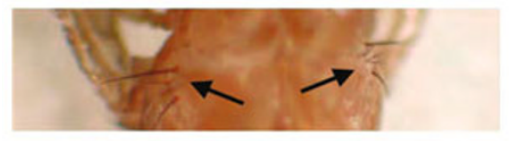

G

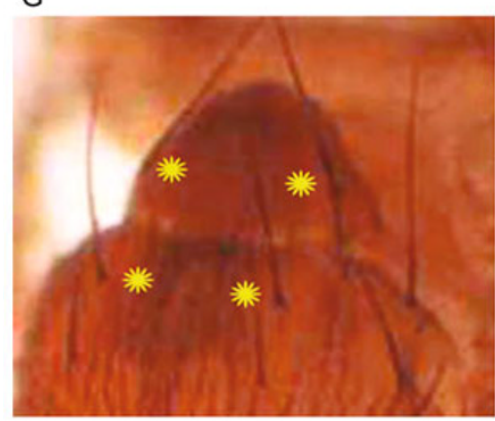

B

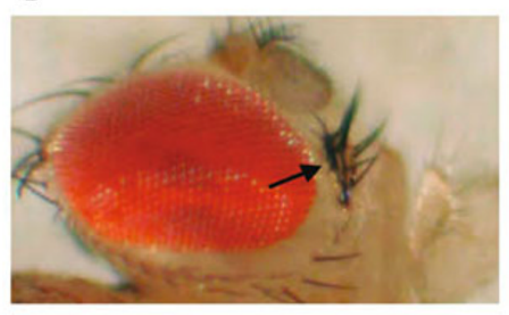

E

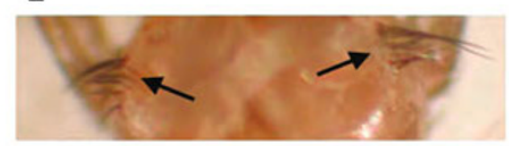

$\mathrm{H}$

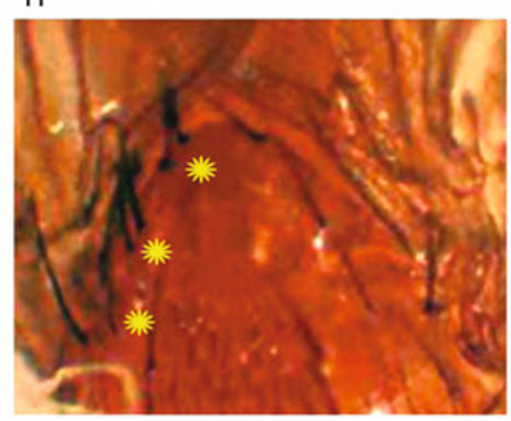

C

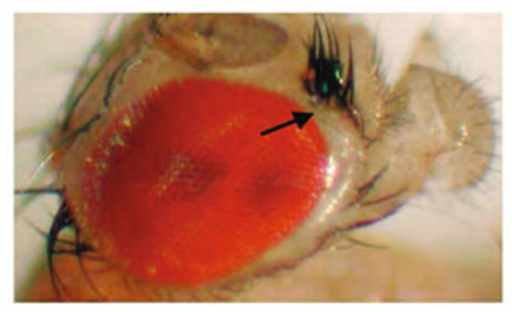

$\mathrm{F}$

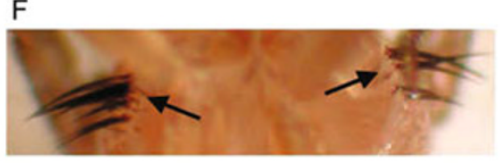

I

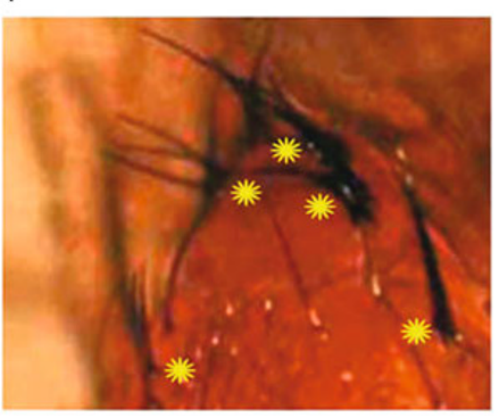

Figure 3. Bristle phenotypes of Psn transgene, and Psn in combination with TSA and PAG. (A) A9-GAL4 > TSA + PAG progeny demonstrated the wild type pattern of evenly spaced bristles anterior to the eye. (B) A9-GAL4 > Psn progeny displayed some ectopic clusters of bristles anterior to the eye (arrow). (C) A9-GAL4 > PSn + PAG + TSA progeny had a more severe phenotype with thicker clusters of ectopic bristles anterior to the eye. (D) 71B-GAL4 > PAG + TSA progeny had wild type sternopleural bristles. (E) 71B-GAL4 > Psn progeny displayed some ectopic sternopleural bristles. (F) 71B-GAL4 > Psn + PAG + TSA progeny displayed a more severe phenotype with greater numbers of ectopic sternopleural bristles. (G) MS1096-GAL4>TSA + PAG progeny were indistinguishable from wild type in terms of the macrochaetae (wild type shown). (H) MS1096-GAL4 > Psn progeny had ectopic macrochaetae that appeared to be in pairs next to the normal macrochaetae. (I) MS1096-GAL4 > Psn + PAG + TSA progeny had numerous macrochaetae clusters.

ectopic bristles increased substantially (Fig. 3C, arrow). A similar effect was observed using the 71B-GAL4 driver. While expression of either TSA or PAG with 71B-GAL4 had no phenotype in the sternopleural bristles (Fig. 3D, wild type shown), Psn expression produced a number of thick ectopic sternopleural bristles (Fig. 3E, arrow). This phenotype was markedly enhanced by co-expression of Psn with TSA and PAG (Fig. 3F, arrow). Finally, similar phenotypes were observed with regard to the scutellar macrochaetae with the MS1096-GAL4 driver. While both wild type flies and those expressing TSA and PAG had four normal macrochaetae (Fig. 3G, yellow stars), expression of the Psn transgene led to an increase in the number of macrochaetae (Fig. 3H). This phenotype was enhanced by co-expression of Psn with TSA and PAG.

\section{The synergism of TSA or PAG with Psn is blocked by activated Notch}

The enhanced loss of Notch-like phenotypes resulting from co-expression of the peroxiredoxin enzymes TSA and PAG with Psn (e.g. thickened veins and increased formation of ectopic sensory organs) suggested that this synergy was mediated by reducing activity of Notch signaling since this pathway is known to be involved in restricting vein thickness, and in cell fate decisions of the sensory organ precursors to limit the number of cells assuming the sensory organ precursor cell fate through lateral inhibition (ArtavanisTsakonas et al., 1999). The overexpression of Psn alone can produce Notch loss-of-function phenotypes, which presumably reflects a dominant negative effect caused by altering the stoichiometry of the multisubunit $\gamma$-secretase complex (Ye et al., 1999). We therefore hypothesized that the synergistic effect we observed with the addition of TSA and PAG was due to exacerbation of this dominant negative effect resulting in a further reduction in Notch signaling. We tested this hypothesis by co-expressing Psn, TSA and PAG with MS1096-GAL4 in flies carrying a dominant activated allele of Notch known as Abruptex ( $N^{A b x}$ ) (Fig. 4). Since $N^{A b x}$ can stimulate Notch signaling in the absence of $\gamma$-secretase, we 
A

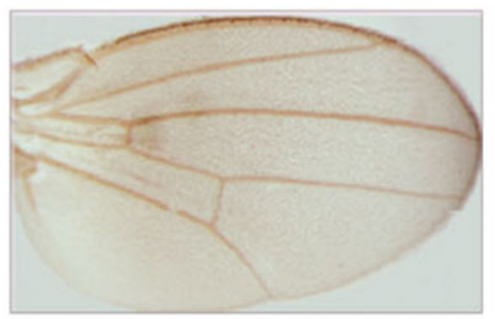

D

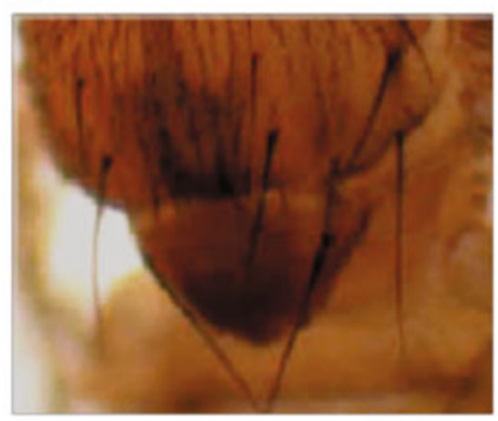

B

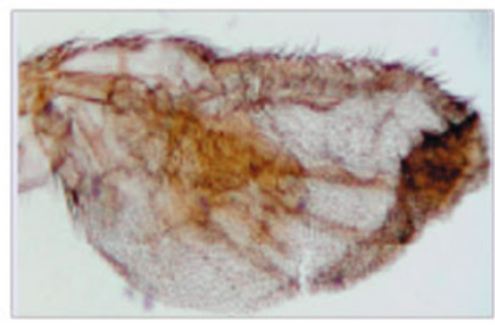

E

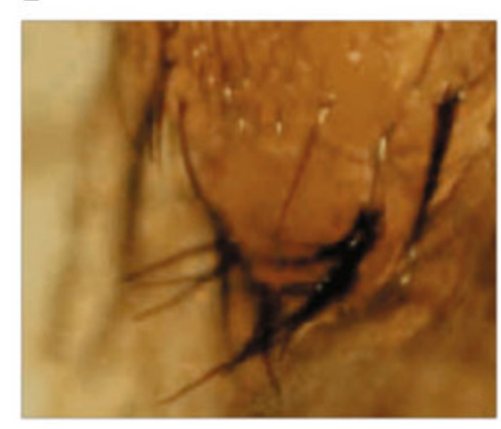

C

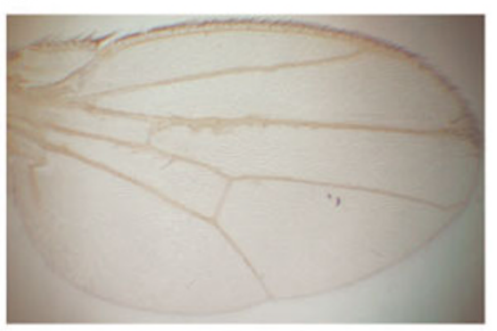

$\mathrm{F}$

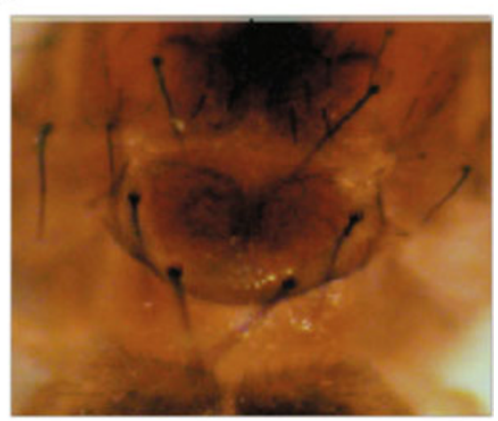

Figure 4. Rescue of Psn + TSA + PAG phenotypes with activated Notch abruptex $\left(\boldsymbol{N}^{A b x}\right)$. (A) The wild type wing. (B) MS1096GAL4 > Psn-UAS + PAG-UAS + TSA-UAS phenotype with severe L3 and L5 wing-vein thickening. (C) $N^{A b x}+$ MS1096-GAL4 > PsnUAS + PAG-UAS + TSA-UAS shows partial rescue of the wing-phenotype with minimal L3 thickening and some L5 thickening. (D) The wild type pattern of macrochaetae on the scutellum. (E) Macrochaetae phenotype observed in the MS1096-GAL4 > Psn-UAS + PAG-UAS + TSA-UAS progeny. (F) Rescue of the phenotype with wild type pattern of macrochaetae in the $N^{A b x}+M S 1096-$ GAL4 > Psn-UAS + PAG-UAS + TSA-UAS progeny.

expected that this allele might rescue the vein and bristle pattern due to expression of Psn with PAG and TSA. Indeed, we found that flies expressing Psn, TSA and PAG in an $N^{A b x}$ background had only residual thickening of the L3 and L5 wing veins (Fig. 4C, compared to wild type in 4A) which was significantly less severe than that observed in response to flies expressing Psn + TSA + PAG (Fig. 4B). In addition, the ectopic scutellar macrochaetae of flies co-expressing Psn, TSA and PAG (Fig. 4E) was nearly completely suppressed by $N^{A b x}$ (Fig. 4F, compared to wild type in 4D). These data support the hypothesis that TSA and PAG act by aggravating the Notch inhibitory effect of Psn overexpression.

\section{DISCUSSION}

The underlying genetic heterogeneity of Alzheimer's disease has made understanding the pathogenesis of the disease difficult without the aid of model genetic organisms. It is clear that plaque formation and oxidative damage are key pathogenetic mechanisms in AD (Smith et al., 2000). The presence of early oxidative stress in mice with Presenilin mutations lends further support for the idea that plaque formation and oxidative damage are connected in $A D(G u$ et al., 2008). However, how these two pathogenetic processes are linked is not known. We have used Drosophila as a model system to explore a protein-protein interaction between presenilin and two peroxiredoxin family members TSA and PAG, which may provide a link between oxidative stress and plaque formation in AD.

There is genetic and biochemical evidence that peroxiredoxin family members play a role in the pathogenesis of AD. There are six peroxiredoxin family members in humans and the evidence for their involvement in $A D$ is summarized in Table 1. Of these genes PRDX1 (PAG) was implicated in physical interaction with human Presenilin-1 and is primarily expressed in glia (Zhou et al., 2002; Hattori and Oikawa, 2007). PRDX2 (TSA) is globally expressed in the brain and has increased expression in the frontal cortex of patients with AD (Krapfenbauer et al., 2003) and is implicated in Parkinson's disease and amyotrophic lateral sclerosis (Boulos et al., 2007). Another family member PRDX6 has been more directly implicated in AD by linkage studies (Liu et al., 2007; Butler et al., 2009). The PRDX6 protein displays markedly increased expression in glia surrounding amyloid plaques in AD brain (Power et al., 2008). Overall these studies support a role for some of these family members in AD pathogenesis. Furthermore, they demonstrate an increase in the expression of PRDX family members in AD brain, an effect which our data suggest could impact the function of Presenilin. These observations suggest our study models a clinically relevant pathogenetic mechanism.

One advantage of the Drosophila wing as an assay system 
Table 1 Genetic and biochemical evidence for a role for PRDX loci in Alzheimer's disease or other human neurodegenerative diseases

\begin{tabular}{|c|c|c|c|c|c|}
\hline $\begin{array}{l}\text { Gene } \\
\text { name }\end{array}$ & Locus & $\begin{array}{l}\text { Protein } \\
\text { subcellular } \\
\text { localization }\end{array}$ & $\begin{array}{l}\text { CNS } \\
\text { expression }\end{array}$ & $\begin{array}{l}\text { Implication in neurodegenerative } \\
\text { disease }\end{array}$ & Reference(s) \\
\hline PRDX1 & $1 \mathrm{p} 34.1$ & $\begin{array}{l}\text { cytoplasm, } \\
\text { melanosome, } \\
\text { mitochondrion, } \\
\text { nucleus }\end{array}$ & $\begin{array}{l}\text { Glial not } \\
\text { neuronal }\end{array}$ & $\begin{array}{l}\text { Neuronal cell death in culture with high expression, } \\
\text { rescued by Presenilin-1 co-expression }\end{array}$ & $\begin{array}{l}\text { Zhou et al., 2002; } \\
\text { Hattori et al., } 2007\end{array}$ \\
\hline PRDX2 & $19 p 13.2$ & $\begin{array}{l}\text { Cytoplasm, } \\
\text { mitochondria }\end{array}$ & Global & $\begin{array}{l}\text { Increased protein expression in the frontal cortex of } \\
\text { patients with Alzheimer's and Parkinson's disease. } \\
\text { Protects neurons in culture from ischemic } \\
\text { and oxidative injury. Inactivation by S-nitrosylation } \\
\text { promotes cell death in Parkinson's disease } \\
\text { Loss of expression in motor neurons } \\
\text { in ALS mouse models }\end{array}$ & $\begin{array}{l}\text { Krapfenbauer et al., 2003; } \\
\text { Boulos et al., 2007; } \\
\text { Fang et al., 2007; } \\
\text { Kato et al., } 2005\end{array}$ \\
\hline PRDX3 & $10 q 26.1$ & $\begin{array}{l}\text { Cytoplasm, } \\
\text { early endosome, } \\
\text { mitochondria }\end{array}$ & Neuronal & $\begin{array}{l}\text { Reduced expression in the frontal cortex of patients } \\
\text { with Parkinson's disease }\end{array}$ & Krapfenbauer et al., 2003 \\
\hline PRDX4 & Xp22.11 & $\begin{array}{l}\text { Cytoplasm, } \\
\text { mitochondria, } \\
\text { extracellular } \\
\text { space }\end{array}$ & Neuronal & Not implicated & N/A \\
\hline PRDX5 & $11 q 13$ & $\begin{array}{l}\text { Cytoplasm, } \\
\text { mitochondria, } \\
\text { peroxisome }\end{array}$ & Neuronal & Not implicated & $\mathrm{N} / \mathrm{A}$ \\
\hline PRDX6 & $1 \mathrm{q} 25.1$ & $\begin{array}{l}\text { Cytoplasm, } \\
\text { lysosome }\end{array}$ & $\begin{array}{l}\text { Glial not } \\
\text { neuronal }\end{array}$ & $\begin{array}{l}\text { Meta-analysis of linkage studies and individual linkage } \\
\text { studies with significant LOD scores at } 1 \mathrm{q} 25 \text {, } \\
\text { Marked increase in protein expression in glia } \\
\text { surrounding neuritic plaques in AD brain }\end{array}$ & $\begin{array}{l}\text { Butler et al., 2009; } \\
\text { Liu et al., 2007; } \\
\text { Power et al., } 2008\end{array}$ \\
\hline
\end{tabular}

is the ability to make use of a wealth of knowledge and an array of genetic tools for analyzing wing development. For example, in this study we observed phenotypes with overexpression of Psn which recapitulated Notch loss-of-function, and we observed a substantial increase in the penetrance and severity of these phenotypes when TSA and PAG were co-expressed with Psn. This led to the hypothesis that TSA and PAG enhance the dominant negative effect of overexpressed Psn. We were able to test whether this enhancement was mediated by the Notch pathway by suppressing this effect with an activated allele of Notch (Fig. 4). Since Psn acts in conjunction with three other components that comprise $\mathrm{Y}$ secretase, it is likely that overexpression of Psn acts in a dominant negative fashion to inhibit Notch signaling by altering the normal stoichiometry of $\mathrm{Y}$-secretase subunits (Struhl and Greenwald, 1999). Our results suggest that association of Psn with TSA or PAG aggravates this effect of Psn overexpression. Interestingly, this seemed to occur with the human peroxiredoxin isoforms in the presence of the Drosophila Psn transgene. While the portion of the PSEN1 protein which interacts with TSA and PAG is only partially conserved with $P s n$, our observations suggest a functionally significant effect across species. Furthermore, we saw an exacerbation of phenotype in response to overexpression of peroxiredoxin antioxidant enzymes, conditions that one might expect to provide oxidative protection. One possible explana- tion for these findings is that by associating with Psn, TSA and PAG further reduce the complete intact $y$-secretase complex. Alternatively, Notch inactivation could be a result of either degradation or abnormal processing of the endogenous presenilin in response to the expression of $P s n$ along with the mammalian transgenes. Either possibility could have obvious disease relevance, as it seems clear that increased expression of these proteins occurs in AD brain (Krapfenbauer et al., 2003; Hattori and Oikawa, 2007), and our data suggest that such an increase in expression could have an impact on Presenilin function and therefore indirectly could influence plaque formation.

While our results support a role for peroxiredoxins PAG and TSA in modifying the Notch inactivation phenotype caused by Psn overexpression, we cannot infer a direct role for these proteins in the processing of amyloid precursor protein, or in the pathogenesis of AD. Loss-of-function studies are needed to determine the mechanism of peroxiredoxin function and whether there is a clear role for endogenous peroxiredoxin in Notch signaling or amyloid cleavage. Further experiments in mammalian cells will be needed to determine whether peroxiredoxin family members are indeed involved in amyloid processing. Nonetheless, we have demonstrated that Drosophila provides an effective system for testing the in vivo relevance of $A D$ related protein-protein interactions. As our wing assay for Psn activity has proven robust in validating a 
suspected protein-protein interaction with Presenilin, it should be amenable to screening for new unknown functionally interacting partners of Psn.

\section{MATERIALS AND METHODS}

\section{Drosophila stocks and transgenic constructs}

The Presenilin transgene was provided by Mark Fortini (Ye and Fortini, 1999). Human TSA and PAG were subcloned into the pUAST vector and injected into $\mathrm{W}$-embryos according to standard procedures (Rubin and Spradling, 1982). Injected larvae were grown and crossed to $w$ - flies with $w+$ selection in the next generation. PAG-UAS insertions were obtained on chromosomes 2 and $X$, while TSA-UAS insertions were obtained on chromosomes 3 and $X$. The following stocks were then generated using recombination (1) w-; +/+; PsnUAS + TSA-UAS. (2) w-; PAG-UAS/CyO; Psn-UAS + TSA-UAS. (3) w-; PAG-UAS/CyO; TSA-UAS. (4) w-; PAG-UAS/CyO; Psn-UAS. (5) Notch Abruptex + MS1096-GAL4; +/+; +/+. (6) MS1096-GAL4 + TSAUAS; +/+; +/+. (7) MS1096-GAL4 + PAG-UAS; +/+; +/+.

\section{Fly mounting}

Adult flies were anesthetized under carbon dioxide and selected for genotype if necessary and stored in isopropanol. Images of Macrochaetae, Sternopleural bristles, and anterior eye bristles were taken on fresh samples in isopropanol. Adult wings were dissected from the thorax and mounted on microscopic slides in Canada balsam. Fly wings were imaged with Nomarski optics.

\section{Statistical analysis}

The penetrance analysis was performed on a series of individual crosses using the 71B-GAL 4 and the transgene stocks. The crosses were performed simultaneously under identical conditions at $29^{\circ} \mathrm{C}$. Penetrance was defined based on phenotypic findings such as ectopic campaniform sensilla, ectopic double row bristles, and ectopic sternopleural bristles. Statistical analysis was performed using Graphpad Prism Software.

\section{ACKNOWLEDGEMENTS}

The authors acknowledge Mark Fortini for providing the Psn transgenic stocks used in this study and Annabelle Guichard for helpful advice.

\section{ABBREVIATIONS}

AD, Alzheimer's disease; PAG, proliferation associated gene (PRDX1); PRDX, Peroxiredoxin; PSEN1, Human Presenilin-1; Psn, Drosophila Presenilin; TSA, Thiol-specific antioxidant (PRDX2); UAS, upstream activator sequence

\section{REFERENCES}

Artavanis-Tsakonas, S., Rand, M.D., and Lake, R.J. (1999). Notch signaling: cell fate control and signal integration in development. Science 284, 770-776.
Bier, E. (2005). Drosophila, the golden bug, emerges as a tool for human genetics. Nat Rev Genet 6, 9-23.

Borchelt, D.R., Thinakaran, G., Eckman, C.B., Lee, M.K., Davenport, F., Ratovitsky, T., Prada, C.M., Kim, G., Seekins, S., Yager, D., et al. (1996). Familial Alzheimer's disease-linked presenilin 1 variants elevate Abeta1-42/1-40 ratio in vitro and in vivo. Neuron 17, 1005-1013.

Boulos, S., Meloni, B.P., Arthur, P.G., Bojarski, C., and Knuckey, N.W. (2007). Peroxiredoxin 2 overexpression protects cortical neuronal cultures from ischemic and oxidative injury but not glutamate excitotoxicity, whereas $\mathrm{Cu} / \mathrm{Zn}$ superoxide dismutase 1 overexpression protects only against oxidative injury. J Neurosci Res 85, 3089-3097.

Brand, A.H., and Perrimon, N. (1993). Targeted gene expression as a means of altering cell fates and generating dominant phenotypes. Development 118, 401-415.

Butler, A.W., Ng, M.Y., Hamshere, M.L., Forabosco, P., Wroe, R., AlChalabi, A., Lewis, C.M., and Powell, J.F. (2009). Meta-analysis of linkage studies for Alzheimer's disease-a web resource. Neurobiol Aging 30, 1037-1047.

Citron, M., Westaway, D., Xia, W., Carlson, G., Diehl, T., Levesque, G., Johnson-Wood, K., Lee, M., Seubert, P., Davis, A., et al. (1997). Mutant presenilins of Alzheimer's disease increase production of 42- residue amyloid beta-protein in both transfected cells and transgenic mice. Nat Med 3, 67-72.

Crowther, D.C., Kinghorn, K.J., Miranda, E., Page, R., Curry, J.A., Duthie, F.A., Gubb, D.C., and Lomas, D.A. (2005). Intraneuronal Abeta, non-amyloid aggregates and neurodegeneration in a Drosophila model of Alzheimer's disease. Neuroscience 132, 123-135.

De Strooper, B., Annaert, W., Cupers, P., Saftig, P., Craessaerts, K., Mumm, J.S., Schroeter, E.H., Schrijvers, V., Wolfe, M.S., Ray, W. J., et al. (1999). A presenilin-1-dependent gamma-secretase-like protease mediates release of Notch intracellular domain. Nature 398, 518-522.

Duff, K., Eckman, C., Zehr, C., Yu, X., Prada, C.M., Perez-tur, J., Hutton, M., Buee, L., Harigaya, Y., Yager, D., et al. (1996). Increased amyloid-beta42(43) in brains of mice expressing mutant presenilin 1. Nature $383,710-713$.

Duffy, J.B. (2002). GAL4 system in Drosophila: a fly geneticist's Swiss army knife. Genesis 34, 1-15.

Fox, N.C., and Schott, J.M. (2004). Imaging cerebral atrophy: normal ageing to Alzheimer's disease. Lancet 363, 392-394.

Gibson, G.E., Zhang, H., Sheu, K.R., and Park, L.C. (2000). Differential alterations in antioxidant capacity in cells from Alzheimer patients. Biochim Biophys Acta 1502, 319-329.

Goate, A., Chartier-Harlin, M.C., Mullan, M., Brown, J., Crawford, F., Fidani, L., Giuffra, L., Haynes, A., Irving, N., James, L., et al. (1991). Segregation of a missense mutation in the amyloid precursor protein gene with familial Alzheimer's disease. Nature 349, 704-706.

Greeve, I., Kretzschmar, D., Tschape, J.A., Beyn, A., Brellinger, C., Schweizer, M., Nitsch, R.M., and Reifegerste, R. (2004). Agedependent neurodegeneration and Alzheimer-amyloid plaque formation in transgenic Drosophila. J Neurosci 24, 3899-3906.

Gu, F., Zhu, M., Shi, J., Hu, Y., and Zhao, Z. (2008). Enhanced oxidative stress is an early event during development of Alzheimerlike pathologies in presenilin conditional knock-out mice. Neurosci 
Lett 440, 44- 48.

Guillen, I., Mullor, J.L., Capdevila, J., Sanchez-Herrero, E., Morata, G., and Guerrero, I. (1995). The function of engrailed and the specification of Drosophila wing pattern. Development 121, 3447-3456.

Haass, C., and De Strooper, B. (1999). The presenilins in Alzheimer's disease-proteolysis holds the key. Science 286, 916-919.

Hattori, F., and Oikawa, S. (2007). Peroxiredoxins in the central nervous system. Subcell Biochem 44, 357-374.

lijima, K., Liu, H.P., Chiang, A.S., Hearn, S.A., Konsolaki, M., and Zhong, Y. (2004). Dissecting the pathological effects of human Abeta40 and Abeta42 in Drosophila: a potential model for Alzheimer's disease. Proc Natl Acad Sci USA 101, 6623-6628.

Krapfenbauer, K., Engidawork, E., Cairns, N., Fountoulakis, M., and Lubec, G. (2003). Aberrant expression of peroxiredoxin subtypes in neurodegenerative disorders. Brain Res 967, 152-160.

Lahiri, D.K., and Greig, N.H. (2004). Lethal weapon: amyloid betapeptide, role in the oxidative stress and neurodegeneration of Alzheimer's disease. Neurobiol Aging 25, 581-587.

Leutner, S., Czech, C., Schindowski, K., Touchet, N., Eckert, A., and Muller, W.E. (2000). Reduced antioxidant enzyme activity in brains of mice transgenic for human presenilin-1 with single or multiple mutations. Neurosci Lett 292, 87-90.

Levy-Lahad, E., Wasco, W., Poorkaj, P., Romano, D.M., Oshima, J., Pettingell, W.H., Yu, C.E., Jondro, P.D., Schmidt, S.D., Wang, K., et al. (1995). Candidate gene for the chromosome 1 familial Alzheimer's disease locus. Science 269, 973-977.

Liu, F., Arias-Vasquez, A., Sleegers, K., Aulchenko, Y.S., Kayser, M., Sanchez-Juan, P., Feng, B.J., Bertoli-Avella, A.M., van Swieten, J., Axenovich, T.I., et al. (2007). A genomewide screen for late-onset Alzheimer disease in a genetically isolated Dutch population. Am J Hum Genet 81, 17-31.

Marcum, J.L., Mathenia, J.K., Chan, R., and Guttmann, R.P. (2005). Oxidation of thiol-proteases in the hippocampus of Alzheimer's disease. Biochem Biophys Res Commun 334, 342-348.

Martins, R.N., Turner, B.A., Carroll, R.T., Sweeney, D., Kim, K.S., Wisniewski, H.M., Blass, J.P., Gibson, G.E., and Gandy, S. (1995). High levels of amyloid-beta protein from S182 (Glu246) familial Alzheimer's cells. Neuroreport 7, 217-220.

McLellan, M.E., Kajdasz, S.T., Hyman, B.T., and Bacskai, B.J. (2003). In vivo imaging of reactive oxygen species specifically associated with thioflavine S-positive amyloid plaques by multiphoton microscopy. J Neurosci 23, 2212-2217.

Mehta, N.D., Refolo, L.M., Eckman, C., Sanders, S., Yager, D., PerezTur, J., Younkin, S., Duff, K., Hardy, J., and Hutton, M. (1998). Increased Abeta42(43) from cell lines expressing presenilin 1 mutations. Ann Neurol 43, 256-258.

Montine, T.J., Neely, M.D., Quinn, J.F., Beal, M.F., Markesbery, W.R., Roberts, L.J. II, and Morrow, J.D. (2002). Lipid peroxidation in aging brain and Alzheimer's disease. Free Radic Biol Med 33, 620-626.

Nowotny, P., Gorski, S.M., Han, S.W., Philips, K., Ray, W.J., Nowotny, V., Jones, C.J., Clark, R.F., Cagan, R.L., and Goate, A.M. (2000). Posttranslational modification and plasma membrane localization of the Drosophila melanogaster presenilin. Mol Cell Neurosci 15, 88-98.

Oyama, F., Sawamura, N., Kobayashi, K., Morishima-Kawashima, M., Kuramochi, T., Ito, M., Tomita, T., Maruyama, K., Saido, T.C.,
Iwatsubo, T., et al. (1998). Mutant presenilin 2 transgenic mouse: effect on an age-dependent increase of amyloid beta-protein 42 in the brain. J Neurochem 71, 313-322.

Patenaude, A., Murthy, M.R., and Mirault, M.E. (2005). Emerging roles of thioredoxin cycle enzymes in the central nervous system. Cell Mol Life Sci 62, 1063-1080.

Perry, G., Nunomura, A., Hirai, K., Zhu, X., Perez, M., Avila, J., Castellani, R.J., Atwood, C.S., Aliev, G., Sayre, L.M., et al. (2002). Is oxidative damage the fundamental pathogenic mechanism of Alzheimer's and other neurodegenerative diseases? Free Radic Biol Med 33, 1475-1479.

Power, J.H., Asad, S., Chataway, T.K., Chegini, F., Manavis, J., Temlett, J.A., Jensen, P.H., Blumbergs, P.C., and Gai, W.P. (2008). Peroxiredoxin 6 in human brain: molecular forms, cellular distribution and association with Alzheimer's disease pathology. Acta Neuropathol 115, 611-622.

Rogaev, E.I., Sherrington, R., Rogaeva, E.A., Levesque, G., Ikeda, M., Liang, Y., Chi, H., Lin, C., Holman, K., Tsuda, T., et al. (1995). Familial Alzheimer's disease in kindreds with missense mutations in a gene on chromosome 1 related to the Alzheimer's disease type 3 gene. Nature 376, 775-778.

Rubin, G.M., and Spradling, A.C. (1982). Genetic transformation of Drosophila with transposable element vectors. Science 218, 348-353.

Scheuner, D., Eckman, C., Jensen, M., Song, X., Citron, M., Suzuki, N., Bird, T.D., Hardy, J., Hutton, M., Kukull, W., et al. (1996). Secreted amyloid beta-protein similar to that in the senile plaques of Alzheimer's disease is increased in vivo by the presenilin 1 and 2 and APP mutations linked to familial Alzheimer's disease. Nat Med 2, 864-870.

Schott, J.M., Price, S.L., Frost, C., Whitwell, J.L., Rossor, M.N., and Fox, N.C. (2005). Measuring atrophy in Alzheimer disease: a serial MRI study over 6 and 12 months. Neurology 65, 119-124.

Schuessel, K., Schafer, S., Bayer, T.A., Czech, C., Pradier, L., MullerSpahn, F., Muller, W.E., and Eckert, A. (2005). Impaired Cu/ZnSOD activity contributes to increased oxidative damage in APP transgenic mice. Neurobiol Dis 18, 89-99.

Selkoe, D.J. (2001). Alzheimer's disease: genes, proteins, and therapy. Physiol Rev 81, 741-766.

Shan, X., Tashiro, H., and Lin, C.L. (2003). The identification and characterization of oxidized RNAs in Alzheimer's disease. J Neurosci 23, 4913-4921.

Sherrington, R., Rogaev, E.I., Liang, Y., Rogaeva, E.A., Levesque, G., Ikeda, M., Chi, H., Lin, C., Li, G., Holman, K., et al. (1995). Cloning of a gene bearing missense mutations in early-onset familial Alzheimer's disease. Nature 375, 754-760.

Smith, C.D., Carney, J.M., Tatsumo, T., Stadtman, E.R., Floyd, R.A., and Markesbery, W.R. (1992). Protein oxidation in aging brain. Ann N Y Acad Sci 663, 110-119.

Smith, M.A., Nunomura, A., Zhu, X., Takeda, A., and Perry, G. (2000). Metabolic, metallic, and mitotic sources of oxidative stress in Alzheimer disease. Antioxid Redox Signal 2, 413-420.

Struhl, G., and Greenwald, I. (1999). Presenilin is required for activity and nuclear access of Notch in Drosophila. Nature 398, 522525.

van de Hoef, D.L., Hughes, J., Livne-Bar, I., Garza, D., Konsolaki, M., and Boulianne, G.L. (2009). Identifying genes that interact with Drosophila presenilin and amyloid precursor protein. Genesis 47, 
246-260.

Van Gassen, G., Annaert, W., and Van Broeckhoven, C. (2000). Binding partners of Alzheimer's disease proteins: are they physiologically relevant? Neurobiol Dis 7, 135-151.

Wolfe, M.S., Xia, W., Ostaszewski, B.L., Diehl, T.S., Kimberly, W.T., and Selkoe, D.J. (1999). Two transmembrane aspartates in presenilin-1 required for presenilin endoproteolysis and gammasecretase activity. Nature 398, 513-517.

Xia, W., Zhang, J., Kholodenko, D., Citron, M., Podlisny, M.B., Teplow, D.B., Haass, C., Seubert, P., Koo, E.H., and Selkoe, D.J. (1997). Enhanced production and oligomerization of the 42residue amyloid beta- protein by Chinese hamster ovary cells stably expressing mutant presenilins. J Biol Chem 272, 7977-7982.

Ye, Y., and Fortini, M.E. (1999). Apoptotic activities of wild-type and Alzheimer's disease-related mutant presenilins in Drosophila melanogaster. J Cell Biol 146, 1351-1364.

Ye, Y., Lukinova, N., and Fortini, M.E. (1999). Neurogenic phenotypes and altered Notch processing in Drosophila Presenilin mutants. Nature 398, 525-529.

Zhou, Y., Zhang, W., Easton, R., Ray, J.W., Lampe, P., Jiang, Z., Brunkan, A.L., Goate, A., Johnson, E.M., and Wu, J.Y. (2002). Presenilin-1 protects against neuronal apoptosis caused by its interacting protein PAG. Neurobiol Dis 9, 126-138. 